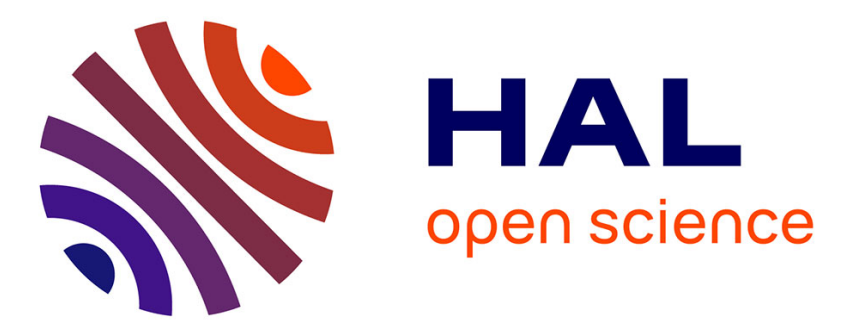

\title{
Effective properties of multiphase composites made of elastic materials with hierarchical structure
}

\author{
Dimitrios Tsalis, Kevin Bonnay, Georges Chatzigeorgiou, Nicolas
}

Charalambakis

\section{To cite this version:}

Dimitrios Tsalis, Kevin Bonnay, Georges Chatzigeorgiou, Nicolas Charalambakis. Effective properties of multiphase composites made of elastic materials with hierarchical structure. Mathematics and Mechanics of Solids, 2015, 10.1177/1081286515612142 . hal-01320767

\section{HAL Id: hal-01320767 \\ https://hal.science/hal-01320767}

Submitted on 24 May 2016

HAL is a multi-disciplinary open access archive for the deposit and dissemination of scientific research documents, whether they are published or not. The documents may come from teaching and research institutions in France or abroad, or from public or private research centers.
L'archive ouverte pluridisciplinaire $\mathbf{H A L}$, est destinée au dépôt et à la diffusion de documents scientifiques de niveau recherche, publiés ou non, émanant des établissements d'enseignement et de recherche français ou étrangers, des laboratoires publics ou privés. 


\title{
Effective properties of multiphase composites made of elastic materials with hierarchical structure
}

\author{
Dimitrios Tsalis $^{* 1}$, Kevin Bonnay ${ }^{2}$, George Chatzigeorgiou ${ }^{2}$ and Nicolas Charalambakis $^{1}$ \\ ${ }^{1}$ Department of Civil Engineering, Aristotle University of Thessaloniki, GR 54124, Thessaloniki, \\ Greece \\ ${ }^{2}$ LEM3-UMR 7239 CNRS, Arts et Metiers ParisTech Metz-Lorraine, 4 Rue Augustin Fresnel 57078 \\ Metz, France
}

\begin{abstract}
In this paper, the analytical solution of the multi - step homogenization problem for multi - rank composites with generalized periodicity made of elastic materials is presented. The proposed homogenization scheme may be combined with computational homogenization for solving more complex microstructures. Three numerical examples are presented, concerning locally periodic stratified materials, matrices with wavy layers and wavy fiber reinforced composites.
\end{abstract}

Keywords

Micromechanics; Generalized periodicity; Multi - step homogenization; Elasticity; Analytical solution.

\section{Introduction}

Natural and manufactured materials may exhibit complex structures with more than one length scale, in a manner that a "hierarchical" structure could be defined (see Fig. 1). This "hierarchy" plays a major role in determining the bulk material properties (Lakes (1993)). Complex micro - architectures can be found in the nature or constructed or accidently obtained in manufacturing processes and the related thermo - electro - mechanical behavior of forming complex structures under different conditions, or the wave propagation in these structures or kinetic techniques and propagation of oscillations are of great technological importance (Jabin \& Tzavaras (2009); Moreno et al. (2014)). Microstructures with periodicity are often characterized by the repetition of the same material element with respect to one or two or three - dimensional coordinate axis. However, there are structures that can not be obtained by the repetition of the same micro - volume. Examples of these structures are the structures with cylidrical periodicity or the locally periodic stratified structures.

One of the simplest composite is a stratified material where the material properties vary only in one direction, called the direction of lamination. This laminate is called rank - 1. In multiple - rank laminates, there is a large difference in the scales of the successive laminations (Fig. 1), which are in different directions (Milton (2002)). More complicated, than rank -1 , locally periodic multilayer structure is the chevron pattern (see Fig.2), usually used as core in sandwich structures 
(Lebee \& Sab (2012)). We refer to the concept of "stratified periodic homogenization", introduced and developed by Bensoussan et al. (1978) and Briane, 1990, 1993, and the concept of "generalized periodicity" presented in Tsalis et al. (2012) and Chatzigeorgiou et al. (2012). Applications of multilayer composites are very common. The human compact bone is a characteristic example of structure that belongs to this caterogy. Generally, it consists of osteons, which are hollow fibers composed of concentric lamellae and pores (Lakes (1993)). The cells of the human heart form fibers nearly parallel to the cardiac wall with orientations varying continuously from the endocardium at the epicardium (Briane, 1990, 1993, Chan \& Leong (2008)). In construction industry, multilayers fiber reinforced composites are very popular since antiquity. Woven fabrics covered by an appropriate matrix in a multilayer structure are used in a wide - range of applications, such as protective materials for military and law enforcement personnel or structural containment of turbine fragments (one of the most widely used applications is in propulsion engine containment systems where engine containment system is typically constructed by wrapping multiple layers of Kevlar @49 around a thin aluminum encasement (Nilakantan et al., 2008; Rajan et al., 2010; Zhu et al., 2014)).

It is known that finite element analysis (FEA) methods are not sufficient to solve heterogeneous problems, since heterogeneities impose restrictions on the size of elements and make too expensive the discretization of heterogeneous structures (Bensoussan et al., 1978; Guedes \& Kikuchi, 1990; Hollister \& Kikuchi, 1992), unless they are combined with micromechanical and/or analytical methods (Hashin, 1983; Kalamkarov \& Kolpakov, 1997; Nemat-Nasser \& Hori, 1999; Aboudi et al., 1999; Nemat-Nasser, 1999; Guinovart-Diaz et al., 2005; Chatzigeorgiou \& Charalambakis, 2005; Love \& Batra, 2006; Chatzigeorgiou et al., 2007; Kalamkarov et al., 2009; Pindera et al., 2009; Nie et al., 2011; Wu et al., 2014; Chatzigeorgiou et al., 2014; Berrehili, 2014; Abd-Alla et al., 2014; Tu \& Pindera, 2014; Savvas et al., 2014; Mahmoud et al., 2014).

Mathematical homogenization (Babuska, 1976a,b; Bensoussan et al., 1978; Sanchez-Palencia, 1978; Francfort, 1983; Francfort \& Murat, 1986; Allaire, 1992; Murat \& Tartar, 1997; Milton, 2002), combined with numerical simulation, finite element or finite volume methods (see for instance Zhigang et al., 2010; Gelebart, 2011; Lee \& Yu, 2011; Nackenhorst et al., 2011; Pindera et al., 2012; Birman et al., 2013; Balokhonov et al., 2014; Katz et al., 2015; ?; Tu \& Pindera, 2014; Freund et al., 2014) remain a rigorous theory for describing heterogeneous materials and structures in engineering applications. Multiscale FEA methods are applied, in which the convergence is improved by defining the basis functions in the following macro-micro way: it is used a coarse mesh for defining the nodal values of the unknown functions and a fine mesh for precomputing the locally periodic oscillating basis functions that depend on the microproperties. So the problem dimension is that of the coarse mesh.

Generalized periodicity - based homogenization theory allows for studying the effective properties in elasticity and generalizes the micromechanics Hill's lemma (see Tsalis et al. (2012) for details). In this paper, we assume that microstructure is periodic with respect to linear or non - linear periodicity surfaces, ordered in different completely distinct scales and forming a multi - rank coated laminate, for the case of linear periodicity (Milton (2002)), or a multi - rank stratified material with generalized (for instance wavy or cylindrical) periodicity. The existence of a sequence of scales of decreasing order allows for performing a succession of homogenization steps, in which the "homogenized" material from every step acts as initial ("heterogeneous") material for the next step. In every step, the homogenization scheme requires the solution of an one - dimensional cell problem with data corresponding to the material properties of the previous step and the volume fractions corresponding to the actual size. We note that in Tsalis et al., 2012, 2013 we presented the solution only of a two-step homogenization and we used trapezoidal rule for the second step. In Section 2 we derive the micro- and macroequation that govern the problem during a homogenization step and present the expression of the effective elastic matrix in terms of the microgeometry expressed by the gradient of the generalized periodicity function. In Section 3 we present the analytical solution of the microequation during a homogenization step, which is necessary in order to compute the effective elastic matrix. In Section 4, three examples are presented, namely a "chevron" pattern, a composite with 
wavy layers and a matrix reinforced by wavy fibers in two directions. The results are crossovered with the results from the Dissipation-Inequality based Periodic Homogenization (DIPH) (see Tsalis et al. (2015)) showing an excellent agreement.

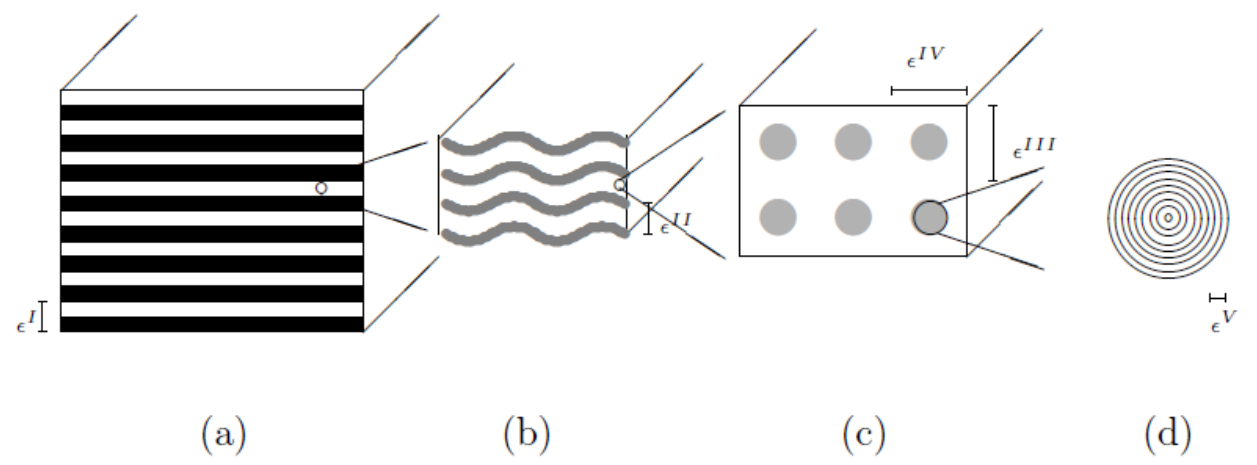

Fig. 1. An example of hierarchical structure, where in every subfigure the structure consists of only two materials $\left(\epsilon^{V} \ll \epsilon^{I V} \sim \epsilon^{I I I} \ll \epsilon^{I I} \ll \epsilon^{I}\right.$, where $\epsilon$ is the heterogeneity parameter (see Section 2 for more details)).

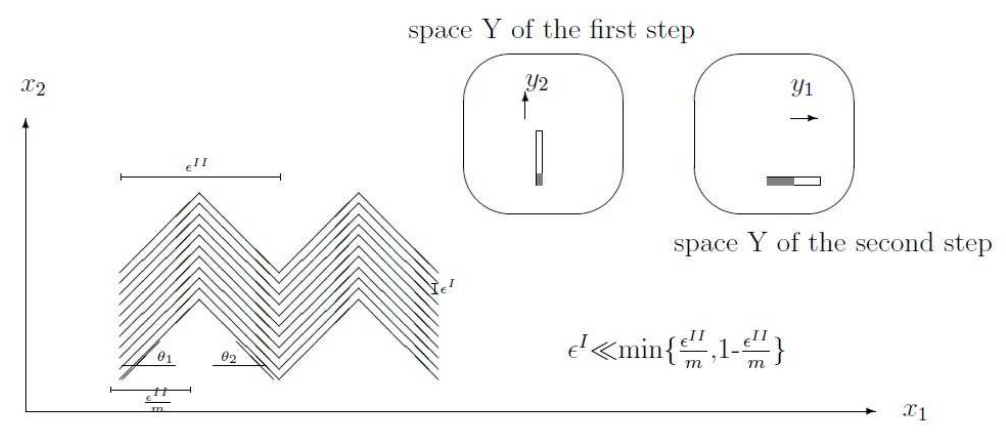

Fig. 2. "Chevron" structure. Two microscale composite with laminate structure, forming angles $\theta_{1}$ and $\theta_{2}$ with $x_{1}$ axis in the macrocoordinate system, the one - dimensional cell in the framework of generalized periodicity in microcoordinate system of the first step and the corresponding of the second step. The material to be homogenized of the second step is a two - phase stratified material.

\section{Definition of the micro- and macroequation problem and the effective elastic matrix}

In mathematical homogenization at least two scales are introduced. The first one is the macroscale denoted by $\boldsymbol{x} \in \Omega$, where $\Omega$ is the volume occupied by the heterogeneous body, at which the heterogeneities, characterized by $\epsilon$, are very small compared to the whole structure. The second one is the microscale denoted by $\frac{\boldsymbol{x}}{\epsilon}$, which is the actual scale for the heterogeneities. At every step of homogenization, the scale is much bigger than the scale of the previous step.

The choice of the RVE is made with respect to the generalized periodicity function $\varrho(x)$ and $Y=\left[0, y_{1}\right] \times\left[0, y_{2}\right] \times\left[0, y_{3}\right]$ is chosen to be the basic cell, where

$$
y=\frac{\underline{\varrho}(\boldsymbol{x})}{\epsilon},
$$

where the parametric equations $\varrho(x)=$ constant describe the surfaces into the structure, with respect to which a material property $\varphi$ is periodic (for more details see Tsalis et al. (2012)). Generally, generalized periodicity function $\varrho$ is a vector valued function (i.e. in the case of (wavy) stratified material, it is simplified to a scalar function while in the case of cylindrical periodicity, it is a two component vector). In the general case, the non-linear periodicity (for instance cylindrical periodicity), if solved by cylindrical coordinates, needs a sufficiently large number of cell problems to be solved. On the contrary, using generalized periodicity, the problem needs the solution of only one cell problem (see Fig. 4). In the case 


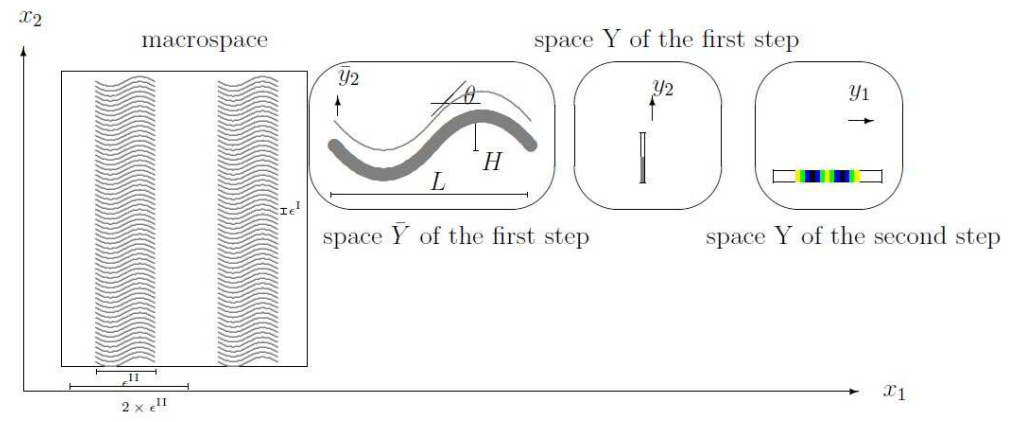

Fig. 3. A typical wavy two - microscale multilayered material. The material to be homogenized of the second step is orthotropic.

of wavy layers, the two-dimensional problem $\bar{Y}$, thanks to the generalized periodicity $\varrho$, becomes one-dimensional (in Fig. 3 the space Y of the first step).

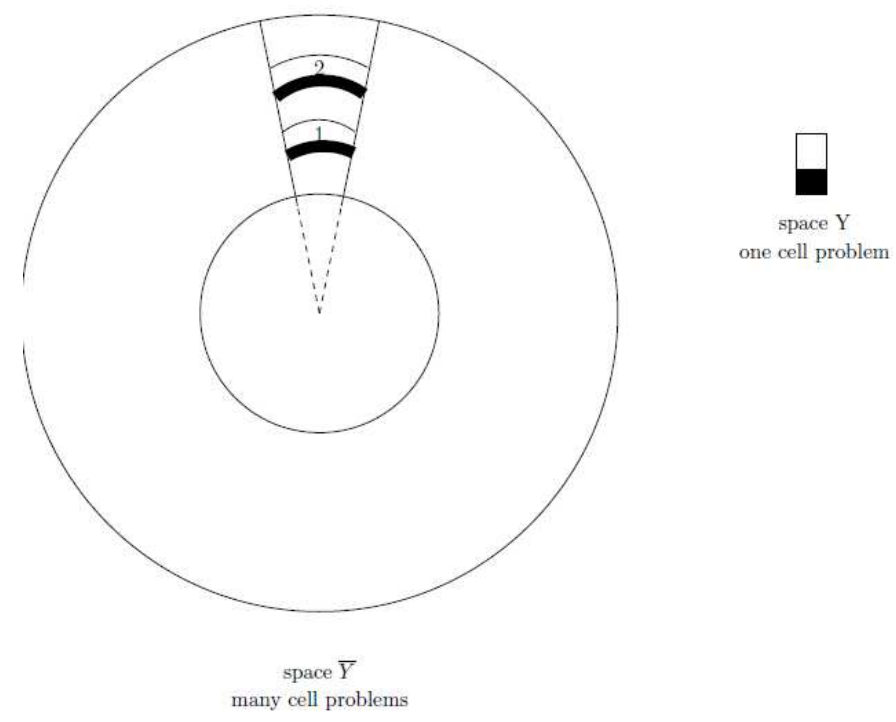

Fig. 4. Using the concept of generalized periodicity function we are able to define a unique cell problem for the whole structure instead of a sufficiently large number of cell problems in different radial positions.

Then, if these surfaces are very close each other forming a microstructure (without necessarily geometric periodicity), the property $\varphi$ is periodic with respect to $\frac{\boldsymbol{\varrho}(\boldsymbol{x})}{\epsilon}$. We write then

$$
\varphi^{\epsilon}(\boldsymbol{x})=\varphi\left(x, \frac{\underline{\varrho}(\boldsymbol{x})}{\epsilon}\right), \quad \text { periodic with respect to } \frac{\boldsymbol{\varrho}(\boldsymbol{x})}{\epsilon}
$$

or

$$
\varphi(\boldsymbol{x}, \boldsymbol{y}), \quad y-\text { periodic. }
$$

The dependence of functions on the microcoordinate is performed (generally in a non - periodic way) via

$$
\overline{\boldsymbol{y}}=\frac{\boldsymbol{x}}{\epsilon} .
$$

We denote field variables $\boldsymbol{\sigma}^{0}, \varepsilon^{0}$ and $\boldsymbol{u}^{1}$ as microscopic variables and $\boldsymbol{\Sigma}, \boldsymbol{E}$ and $\boldsymbol{u}^{0}$ as the macroscopic variables where the macroscopic quantities depend only on the macrocoordinate $\boldsymbol{x}$. Both classes of deformation fields are related to the unit cell located at $\boldsymbol{x}$. Away from the boundaries $\partial \Omega$, stress and strain fields conform at the microlevel to the generalized 
periodicity conditions:

$$
\boldsymbol{\sigma}^{0}, \varepsilon^{0} \text { are } Y-\text { periodic functions of } \boldsymbol{y} \text {. }
$$

This assumption is equivalent to the assertion that the stress belongs to the space of microscopically self - equilibrated fields.

The actual displacement $\boldsymbol{u}^{0}$ within $Y$ located at $\boldsymbol{x}$ has two properties: it is oscillating and it has a generalized periodicity. We assume that $\boldsymbol{u}^{0}$ can be expressed as a sum of a linear and a periodic part (Tartar, 1978; Suquet, 1985; Tsalis et al., 2012)

$$
u_{i}^{0}(\boldsymbol{x}, \overline{\boldsymbol{y}}, \boldsymbol{y})=E_{i j} \bar{y}_{j}+u_{i}^{1}
$$

where

$$
u_{i}^{1}=u_{i}^{1}(\boldsymbol{x}, \boldsymbol{y}),
$$

is periodic with respect to $y$. We remind that the difference with Suquet (1985) is that, there, the function $u_{i}^{1}$ is periodic with respect to the microvariable $\overline{\boldsymbol{y}}$, while here it is periodic only with respect to the generalized periodicity (Tsalis et al. (2012)).

Microstrain is defined from (6) with respect to the microcoordinate,

$$
\varepsilon_{i j}^{0}=\operatorname{sym}\left(\frac{\partial u_{i}}{\partial \bar{y}_{j}}\right)=\frac{1}{2}\left(\frac{\partial u_{i}}{\partial \bar{y}_{j}}+\frac{\partial u_{j}}{\partial \bar{y}_{i}}\right)
$$

while the macrostrain is defined with respect to the macrocoordinate,

$$
E_{i j}=\operatorname{sym}\left(\frac{\partial u_{i}^{0}}{\partial x_{j}}\right)=\frac{1}{2}\left(\frac{\partial u_{i}^{0}}{\partial x_{j}}+\frac{\partial u_{j}^{0}}{\partial x_{i}}\right) .
$$

It was prooved (Tsalis et al. (2013)) that

$$
\left\langle\varepsilon_{i j}^{0}\right\rangle=E_{i j} .
$$

We consider a rank - 1 composite made of elastic material exhibiting periodicity with respect to the vector valued function $\varrho(x)$. Two main hypotheses hold. The first is the perfect bonding among layers of the constituents (see in Berrehili (2014) the effect of debonded fibers of a composite under traction). The second one is that there is no cracking in the structure. The elastic coefficients are assumed of the form,

$$
\begin{gathered}
C_{i j k h}(\boldsymbol{y})=C_{i j k h}\left(\frac{\underline{\varrho}(\boldsymbol{x})}{\epsilon}\right), \quad C_{i j k h}(\boldsymbol{y}) \quad Y \text {-periodic, } \quad C_{i j k h} \in L^{\infty}\left(\mathbb{R}^{m}\right), \\
\varrho \in C^{2}\left(\mathbb{R}^{m}\right), \quad m \leq 3, \\
C_{i j k h} \xi_{i j} \xi_{k h} \geq \lambda \xi_{i j} \xi_{i j}, \forall \xi \text { symmetric, } C_{i j k h}=C_{j i k h}=C_{i j h k}=C_{k h i j}, \\
\sum_{i=1}^{n}\left(\sum_{k=1}^{m} \frac{\partial \varrho_{k}(\boldsymbol{x})}{\partial x_{i}} \eta_{k}\right)^{2} \geq \beta \sum_{k=1}^{n} \eta_{k}^{2}, \quad \forall \boldsymbol{\eta} .
\end{gathered}
$$

We note that, at every scale $\frac{1}{\epsilon}$, the generalized periodicity is related only to the actual scale. The sequentially formed composite allows for a succession of homogenization steps based on one-dimensional cell problems. We note that generalized 
periodicity is the ingredient used to reduce the dimension of 2-D or 3-D cell problems in terms of cartesian coordinates (needing a FEM application), to the one-dimensional cell problems mentioned above, that can be solved analytically with smaller computational cost. See also the second example of Section 4.

We study the elasticity problem

$$
\begin{gathered}
\frac{\partial \sigma_{i j}^{\epsilon}}{\partial x_{j}}+f_{i}^{\epsilon}(x)=0 \text { in } \Omega, \\
u_{i}^{\epsilon}=0 \text { on } S_{d}, \\
\sigma_{i j}^{\epsilon} n_{j}=t_{i}(x) \text { on } S_{t}, \\
\sigma_{i j}^{\epsilon}(x)=C_{i j k h}^{\epsilon}(x) \varepsilon_{k h}\left(u^{\epsilon}\right),
\end{gathered}
$$

where $\boldsymbol{\varepsilon}(\boldsymbol{u})=\frac{1}{2}\left(\nabla \boldsymbol{u}+{ }^{t} \nabla \boldsymbol{u}\right)$ is the infinitesimal strain tensor. By putting

$$
V=\left\{\boldsymbol{v} \in\left(H^{1}(\Omega)\right)^{3}|\boldsymbol{v}|_{S_{d}}=0\right\}
$$

where $\left.v\right|_{S_{d}}$ is the value of $v$ on the boundary $S_{d}$, the equations of equilibrium (15), in weak formulation, are written

$$
\int_{\Omega} \nabla_{j} \sigma_{i j}^{\epsilon} \omega_{i} d \Omega+\int_{\Omega} f_{i}^{\epsilon} \omega_{i} d \Omega=0, \quad \forall \omega_{i} \in V
$$

We recall that $H^{1}$ stands for the Sobolev space of $L^{2}$ - functions having $L^{2}$ - derivatives.

The dependence of the solution $\boldsymbol{u}^{\epsilon}$ in both the macroscopic and the microscopic levels justifies the assumption that the displacement and the related quantities (stress and strains) can be expressed as asymptotic expansions in terms of the microstructural parameter.

We apply the asymptotic expansion homogenization technique and we conclude (Tsalis et al. (2012)) that the microstress satisfies the equation of equilibrium,

$$
\frac{\partial \sigma_{i j}^{0}}{\partial \bar{y}_{j}}=0
$$

or

$$
\frac{\partial \sigma_{i j}^{0}}{\partial y_{m}} \frac{\partial \varrho_{m}}{\partial x_{j}}=0
$$

or

$$
-\int_{Y} C_{i j l m} K_{l m}\left(w^{k h}\right) K_{k h}(\omega) d Y=\nabla_{j} \varrho_{l} \int_{Y} \nabla_{y l} C_{i j k h} \omega_{i} d Y,
$$

where $K$ is the symmetric matrix,

$$
K(\boldsymbol{u})=\frac{1}{2}\left(\nabla_{y} \boldsymbol{u} \nabla \boldsymbol{\varrho}+{ }^{t}\left(\nabla_{y} \boldsymbol{u} \nabla \boldsymbol{\varrho}\right)\right)
$$

The macrostress is defined as the mean value of the microstress over the unit cell,

$$
\left\langle\sigma_{i j}^{0}\right\rangle=\Sigma_{i j}
$$

In Tsalis et al. (2013), it is shown that the Mandel - Hill's lemma holds,

$$
\left\langle\sigma_{i j}^{0} \varepsilon_{i j}^{0}\right\rangle=\Sigma_{i j} E_{i j}
$$


while the effective elastic matrix is given by

$$
C_{i j \phi \theta}^{e f f}=\left\langle C_{i j \phi \theta}+C_{i j m n} \frac{\partial w_{m}^{\phi \theta}}{\partial y} \frac{\partial \varrho}{\partial x_{n}}\right\rangle,
$$

or by using Voigt notation for $\mathrm{i}=1, \mathrm{k}=1, \mathrm{l}=6$ and $\mathrm{m}=5$, for $\mathrm{i}=2, \mathrm{k}=6, \mathrm{l}=2$ and $\mathrm{m}=4$, for $\mathrm{i}=3, \mathrm{k}=5, \mathrm{l}=4$ and $\mathrm{m}=3$

$$
C_{p \boldsymbol{a}}^{e f f}=\left\langle C_{p \boldsymbol{a}}+\sum_{i=1}^{3}\left(C_{p k} \frac{\partial \varrho}{\partial x_{1}}+C_{p l} \frac{\partial \varrho}{\partial x_{2}}+C_{p m} \frac{\partial \varrho}{\partial x_{3}}\right) \frac{\partial w_{i}^{\boldsymbol{a}}}{\partial y}\right.
$$

In (26) and (27), $\langle\ldots\rangle$ is the mean value operator. In (27) we use Voigt notation for $p$ instead of $i j$ and $\boldsymbol{a}$ instead of $\phi \theta$. The gradient of the generalized periodicity function enters the effective coefficients, reflecting the microstructure properties at the actual scale $\epsilon$. If there exists a next scale, the effective coefficients (28), obtained from the previous step, act as "heterogeneous" material coefficients of the next homogenization step. In the next section, the partial derivatives of elastic correctors are computed through the solution of the microequation (21).

\section{The solution of the microequation}

The cell equation (21) is written as

$$
\frac{\partial}{\partial y_{l}}\left(\frac{\partial \varrho_{l}}{\partial x_{j}} C_{i j k z} \frac{\partial \varrho_{m}}{\partial x_{z}} \frac{\partial w_{k}^{\phi \theta}}{\partial y_{m}}+\frac{\partial \varrho_{l}}{\partial x_{j}} C_{i j \phi \theta}\right)=0
$$

with respect to the unknowns $w_{l}^{k h}$ (where the indices $l, m$ take the value 1 , the indices $i, k$ take the values $1,2,3$ and the indices $j, z$ take the values 1,2 ) under appropriate periodicity and continuity conditions.

By expanding (29) for $l, m, j, z, k$,

$$
\begin{gathered}
\frac{\partial}{\partial y}\left(\frac{\partial \varrho}{\partial x_{1}} C_{i 1 \phi \theta}+\frac{\partial \varrho}{\partial x_{2}} C_{i 2 \phi \theta}\right)+ \\
\sum_{j=1}^{3} \frac{\partial}{\partial y}\left(\frac{\partial w_{j}^{\phi \theta}}{\partial y}\left(\frac{\partial \varrho}{\partial x_{1}} \frac{\partial \varrho}{\partial x_{1}} C_{i 1 j 1}+\frac{\partial \varrho}{\partial x_{1}} \frac{\partial \varrho}{\partial x_{2}} C_{i 1 j 2}+\frac{\partial \varrho}{\partial x_{2}} \frac{\partial \varrho}{\partial x_{1}} C_{i 2 j 1}+\frac{\partial \varrho}{\partial x_{2}} \frac{\partial \varrho}{\partial x_{2}} C_{i 2 j 2}\right)\right)=0 .
\end{gathered}
$$

Putting

$$
a_{j}^{i}\left(x_{1}, x_{2}\right)=\frac{\partial \varrho}{\partial x_{1}} \frac{\partial \varrho}{\partial x_{1}} C_{i 1 j 1}+\frac{\partial \varrho}{\partial x_{1}} \frac{\partial \varrho}{\partial x_{2}} C_{i 1 j 2}+\frac{\partial \varrho}{\partial x_{2}} \frac{\partial \varrho}{\partial x_{1}} C_{i 2 j 1}+\frac{\partial \varrho}{\partial x_{2}} \frac{\partial \varrho}{\partial x_{2}} C_{i 2 j 2},
$$

for $\mathrm{j}=1,2$ and 3 , equation (30) is written

$$
\frac{\partial}{\partial y}\left(\frac{\partial \varrho}{\partial x_{1}} C_{i 1 \phi \theta}+\frac{\partial \varrho}{\partial x_{2}} C_{i 2 \phi \theta}+a_{1}^{i} \frac{\partial w_{1}^{\phi \theta}}{\partial y}+a_{2}^{i} \frac{\partial w_{2}^{\phi \theta}}{\partial y}+a_{3}^{i} \frac{\partial w_{3}^{\phi \theta}}{\partial y}\right)=0 .
$$

Equations (32) using Voigt notation for the free index $i=1,2,3$ and by substitute $\phi \theta$ by $\boldsymbol{a}$ for $\boldsymbol{a}=1,2,3,4,5,6$, are written

$$
\frac{\partial}{\partial y}\left(\frac{\partial \varrho}{\partial x_{1}} C_{k \boldsymbol{a}}+\frac{\partial \varrho}{\partial x_{2}} C_{l \boldsymbol{a}}+a_{1}^{i} \frac{\partial w_{1}^{\boldsymbol{a}}}{\partial y}+a_{2}^{i} \frac{\partial w_{2}^{\boldsymbol{a}}}{\partial y}+a_{3}^{i} \frac{\partial w_{3}^{\boldsymbol{a}}}{\partial y}\right)=0,
$$

for $\mathrm{i}=1, \mathrm{k}=1$ and $\mathrm{l}=6$, for $\mathrm{i}=2, \mathrm{k}=6$ and $\mathrm{l}=2$, for $\mathrm{i}=3, \mathrm{k}=5$ and $\mathrm{l}=4$.

Intergate equations (33) for $y$, 


$$
{ }^{\text {(mat) }}\left(a_{1}^{i} \frac{\partial w_{1}^{\boldsymbol{a}}}{\partial y}\right)+{ }^{\text {(mat) }}\left(a_{2}^{i} \frac{\partial w_{2}^{\boldsymbol{a}}}{\partial y}\right)+{ }^{\text {(mat) }}\left(a_{3}^{i} \frac{\partial w_{3}^{\boldsymbol{a}}}{\partial y}\right)={ }^{\text {(mat) }} \operatorname{con}_{i}^{\boldsymbol{a}}-{\frac{\partial \varrho}{\partial x_{1}}}^{\text {(mat) }} C_{k \boldsymbol{a}}-{\frac{\partial \varrho}{\partial x_{2}}}^{\text {(mat) }} C_{l \boldsymbol{a}},
$$

for $\mathrm{i}=1, \mathrm{k}=1$ and $\mathrm{l}=6$, for $\mathrm{i}=2, \mathrm{k}=6$ and $\mathrm{l}=2$, for $\mathrm{i}=3, \mathrm{k}=5$ and $\mathrm{l}=4$ and where ${ }^{(\mathrm{mat})} \operatorname{con}_{3}^{a}$ are the basic unknowns.

Equations (34) are written in matrix form

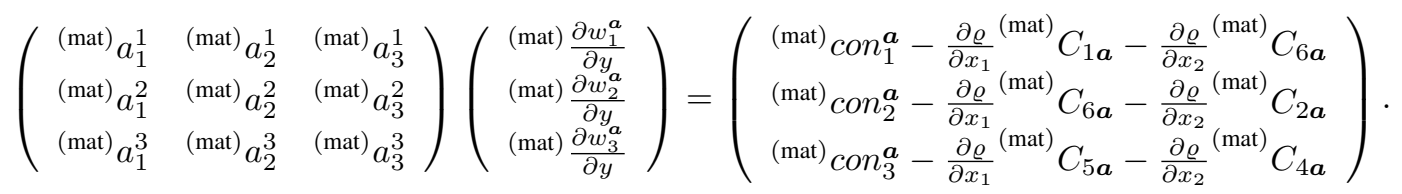

Solving with respect to ${ }^{\text {(mat) }} \frac{\partial w_{1}^{a}}{\partial y}$, (mat) $\frac{\partial w_{2}^{a}}{\partial y}$ and ${ }^{\text {(mat) }} \frac{\partial w_{3}^{a}}{\partial y}$ and intergating for $y$ gives

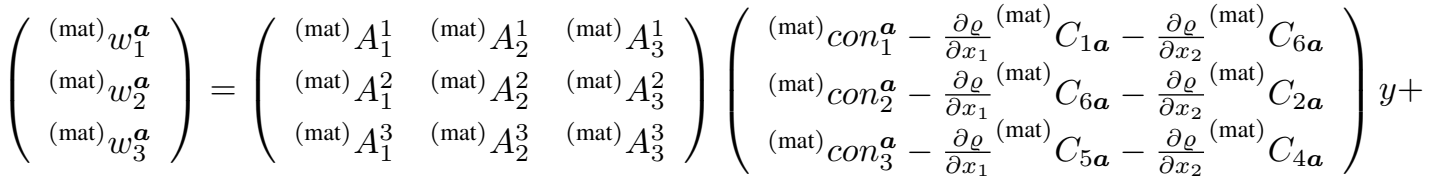

$$
\begin{aligned}
& \left(\begin{array}{c}
{ }^{(m a t)} \operatorname{con}_{1}^{* a} \\
{ }^{(\mathrm{mat})} \operatorname{con}_{2}^{* a} \\
{ }^{(\mathrm{mat})} \operatorname{con}_{3}^{* a}
\end{array}\right)
\end{aligned}
$$

where matrices ${ }^{(\mathrm{mat})} A_{j}^{i}$ are the inverse of the matrices ${ }^{(\mathrm{mat})} a_{j}^{i}$ while the constants ${ }^{(\mathrm{mat})} \operatorname{con}_{1}^{* \boldsymbol{a}},{ }^{\text {(mat) }} \operatorname{con}_{2}^{* \boldsymbol{a}}$ and ${ }^{\text {(mat) }} \operatorname{con}_{3}^{* a}$ are computed by the last equation using the boundary and periodicity conditions of the problem.

The periodicity conditions for the first and the last material in the unit cell read

$$
\text { at } y=0, \quad{ }^{(1)} w_{k}^{\phi \theta}=0 \text { and at } y=1, \quad{ }^{(n)} w_{k}^{\phi \theta}=0 .
$$

The continuity conditions at the material interfaces in the unit cell read

$$
\left[\left|w_{k}^{\phi \theta}\right|\right]=0, \quad\left[\left|\frac{\partial \varrho}{\partial x_{j}} C_{i j l m} \frac{\partial \varrho}{\partial x_{m}} \frac{\partial w_{l}^{k h}}{\partial y}+\frac{\partial \varrho}{\partial x_{j}} C_{i j \phi \theta}\right|\right]=0
$$

where $[|\ldots|]$ denotes the difference at both sides of an interface. The second equation (36) is equivalent to

$$
\left[\left|\frac{\partial \varrho}{\partial x_{j}} \sigma_{i j}^{0}\right|\right]=0
$$

From equations (34) and (37) one obtains that ${ }^{(m a t)} \operatorname{con}_{i}^{a}$ is independent of $y$ and take the same value in every material.

In general, for a $n$-phase composite structure, a system of $18(n-1)$ equations with 18 basic unknowns and 18( $n-2)$ auxialliary unknowns solves the cell problem

$$
\tilde{\boldsymbol{C}} \tilde{\boldsymbol{U}}=\tilde{\boldsymbol{S}}
$$

where the matrix of coefficients $\tilde{\boldsymbol{C}}$, the matrix of unknowns $\tilde{\boldsymbol{U}}$ and the constant matrix $\tilde{\boldsymbol{S}}$ are shown in Appendix A, Tables 19, 20 and 21.

The novelty of this paper is the introduction of an automated process in the form of a "code" (Fig. 5) with analytical expressions for the effective stiffness of a multiphase material with hierarchical structure, while in Tsalis et al. (2012) the simple case of two phases is treated. The present "code" allows for passing easier from the finer scale to the coarser scale 
due to the fact that the result of the coarser scale is the input data of the finer scale. Also, there is no need for $\varrho$ to be the same for all scales, giving the code flexibility.

2-D. 3-D

Input/Output data from

finer/coarser scale using FEM
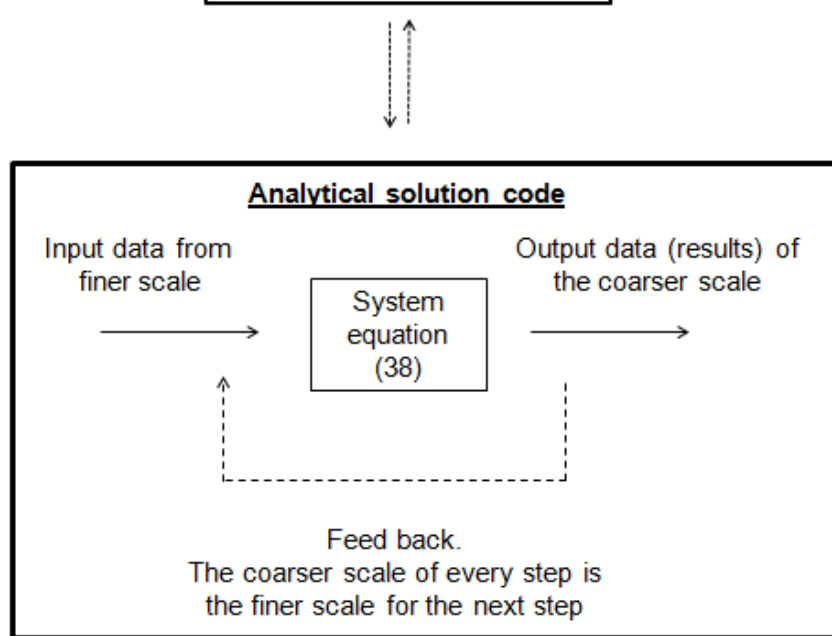

Fig. 5. A schematic representation of the "code".

The case of wavy layered materials is a good example of a structure exhibiting generalized periodicity. In the coordinate system $\boldsymbol{x}$, this problem needs a two-dimensional cell problem formulation. This two-dimensional cell problem can be solved only by FEA application. On the contrary, by considering that the structure exhibits periodicity with respect to the wavy surface, the problem can be formulated as one-dimensional cell problem.

In many cases, the first step of homogenization procedure requires the solution of a three - dimensional cell problem. In this case, cell equations form a system of three partial equations (for illustration purposes, we show only the first equation in Appendix B, the two others resulting in a similar manner). Contrarily to the one - dimensional problem that we have dealt with up to now, this problem has no analytical solution. In order to solve that kind of problems we use FEA commercial softwares, such as DS Simulia Abaqus. By using DS Simulia Abaqus we can solve it completely, but in this case we should take under consideration the computational cost. As an alternatice solution, in this paper we combine the commercial software with the homogenization method mentioned before, following a two - step strategy. In Fig. 1 (a), (b) and (d) the problem is one-dimensional and can be solved using the solution that is presented in this paper. In subfigure (c) the problem is two-dimensional, whithout an analytical solution, and a FEA method in required. The combination of analytical solutions with FEA solution leads to the global problem solution. It should be noticed that despite the fact that generalized periodicity function $\varrho$ is not the same in subfigures (a), (b) and (d), we are able to use the same code due to the fact that this code is parametrized in terms, among other, of the function $\varrho$. This homogenization scheme will be clarified in the third example of the next section.

\section{Numerical examples}

In this section we present three examples of multi - step homogenization: the first example is characterized by a linear generalized periodicity, while the second and the third examples exhibit a non - linear gereralized periodicity. 


\section{1. "Chevron" structure}

The first example is the two - dimensional, two - phase, second - rank laminate shown in Fig. 2. As mentioned, the widths $\frac{\epsilon^{I I}}{2}$ of the slabs should be much larger than the thicknesses of the layers within each slab. The layers within each slab form angles $\theta_{1}$ and $\theta_{2}$, not necessarly equal. The case $\theta_{1}=30^{\circ}, \theta_{2}=60^{\circ}$ is considered. The constituents are assumed isotropic with mechanical properties shown in Tables 1 and 2.

In order to solve this problem we will follow the procedure of two - step homogenization. According to this procedure, the problem will be solved first to the direction normal to the layers of the initial composite and as a result we will obtain a "new intermediate" composite. Next we will apply the same procedure along $x_{1}$ axis for the constituents of the "new intermediate" composite, resulting from the first step. It is obvious that two generalized functions are required. The generalized periodicity function for the first step of the homogenization is $\varrho^{I}=x_{2}-x_{1} \tan \theta$ with gradients $\frac{\partial \varrho^{I}}{\partial x_{1}}=-\tan \theta$ and $\frac{\partial \varrho^{I}}{\partial x_{2}}=1$. The generalized periodicity function for the second step is $\varrho^{I I}=x_{1}$ with gradients $\frac{\partial \varrho^{I I}}{\partial x_{1}}=1$ and $\frac{\partial \varrho^{I I}}{\partial x_{2}}=0$.

The unit cell of the first step of homogenization (parameter of heterogeneity $\epsilon^{I}$ ) consists of $90 \%$ metal and $10 \%$ ceramic, while the volume fractions for the second step depend on the geometry and are depicted in Table 3 . We compute the effective elastic matrix from equation (28) using a $M A T L A B$ code that we have developed.

This is the simplest case since in both steps only two materials (the initial constituents in the first step and the results of the first step as constituents in the second step) are used.

Table 1. "Chevron" structure. Mechanical properties of constituents (Khatam \& Pindera, 2009).

\begin{tabular}{|c|c|c|}
\hline Property & Metal & Ceramic \\
\hline \hline Young Modulus (GPa) & 72.400 & 420.000 \\
\hline Poisson Ratio & 0.330 & 0.250 \\
\hline
\end{tabular}

Table 2. "Chevron" structure. Elastic (symmetric) matrix coefficients of the two constituents (in GPa).

\begin{tabular}{|c|c|c|}
\hline Mechanical Properties & Metal & Ceramic \\
\hline \hline$C_{11}, C_{22}, C_{33}$ & 107.271 & 504.000 \\
\hline$C_{12}, C_{13}, C_{23}$ & 52.835 & 168.000 \\
\hline$C_{44}, C_{55}, C_{66}$ & 27.218 & 168.000 \\
\hline$C_{14}, C_{15}, C_{16}$ & 0 & 0 \\
\hline$C_{24}, C_{25}, C_{26}$ & 0 & 0 \\
\hline$C_{34}, C_{35}, C_{36}$ & 0 & 0 \\
\hline
\end{tabular}

Table 3. "Chevron" structure. Volume fractions of constituents of the second step of homogenization.

\begin{tabular}{|c|c|c|}
\hline $\begin{array}{c}\text { Geometry } \\
\left(\theta_{1} \text { vs } \theta_{2} \text { angle }\right)\end{array}$ & $\begin{array}{c}\text { Phase } 1 \text { volume fraction } \\
\left(\theta_{1} \text { angle }\right)\end{array}$ & $\begin{array}{c}\text { Phase } 2 \text { volume fraction } \\
\left(\theta_{2} \text { angle }\right)\end{array}$ \\
\hline 30 vs 60 & $75.000 \%$ & $25.000 \%$ \\
\hline
\end{tabular}

The results of the first step of homogenization are depicted in Tables 4 and 5, while the effective elastic matrix for the whole unit cell is depicted in Table 7. Comparing Table 7 with Table 8, we conclude that the results of the present approach are in very good agreement with the results of the DIPH (see Tsalis et al. (2015)).

From Table 7 it is obvious that, despite the fact that the composite is made of isotropic elastic materials, it behaves like a monoclinic material. In other words, as it is expected, homogenization causes anisotropy. 
Table 4. "Chevron" structure. Effective elastic matrix for $\theta_{1}=30^{\circ}$ from the first step of homogenization (in GPa).

$$
C^{\text {eff I } \theta_{1}}=\left(\begin{array}{cccccc}
131.579 & 61.306 & 60.210 & 0 & 0 & 9.403 \\
61.306 & 117.610 & 57.067 & 0 & 0 & 2.694 \\
60.210 & 57.067 & 144.373 & 0 & 0 & 2.722 \\
0 & 0 & 0 & 32.605 & 5.018 & 0 \\
0 & 0 & 0 & 5.018 & 38.399 & 0 \\
9.403 & 2.694 & 2.722 & 0 & 0 & 35.518
\end{array}\right)
$$

Table 5. "Chevron" structure. Effective elastic matrix for $\theta_{2}=60^{\circ}$ from the first step of homogenization (in GPa).

$$
C^{\text {eff I } \theta_{2}}=\left(\begin{array}{cccccc}
117.610 & 61.306 & 57.067 & 0 & 0 & -2.694 \\
61.306 & 131.579 & 60.210 & 0 & 0 & -9.403 \\
57.067 & 60.210 & 144.373 & 0 & 0 & -2.722 \\
0 & 0 & 0 & 38.399 & -5.018 & 0 \\
0 & 0 & 0 & -5.018 & 32.605 & 0 \\
-2.694 & -9.403 & -2.722 & 0 & 0 & 35.518
\end{array}\right)
$$

Table 6. "Chevron" structure. Effective elastic matrix for $\theta_{2}=30^{\circ}$ from the first step of homogenization (in GPa).

$$
C^{\text {eff II } \theta_{2}}=\left(\begin{array}{cccccc}
131.579 & 61.306 & 60.210 & 0 & 0 & -9.403 \\
61.306 & 117.610 & 57.067 & 0 & 0 & -2.694 \\
60.210 & 57.067 & 144.373 & 0 & 0 & -2.722 \\
0 & 0 & 0 & 32.605 & -5.018 & 0 \\
0 & 0 & 0 & -5.018 & 38.399 & 0 \\
-9.403 & -2.694 & -2.722 & 0 & 0 & 35.518
\end{array}\right)
$$

Table 7. "Chevron" structure, $\theta_{1}=30^{\circ}, \theta_{2}=60^{\circ}$. Effective elastic matrix from the second step of homogenization by the proposed method (in GPa).

$$
C^{\text {eff I }}=\left(\begin{array}{cccccc}
127.017 & 60.536 & 59.010 & 0 & 0 & 6.119 \\
60.536 & 120.330 & 57.506 & 0 & 0 & -0.328 \\
59.010 & 57.503 & 144.202 & 0 & 0 & 1.303 \\
0 & 0 & 0 & 33.499 & 2.189 & 0 \\
0 & 0 & 0 & 2.189 & 36.766 & 0 \\
6.119 & -0.328 & 1.303 & 0 & 0 & 35.291
\end{array}\right)
$$

Table 8. "Chevron" structure, $\theta_{1}=30^{\circ}, \theta_{2}=60^{\circ}$. Effective elastic matrix by DIPH (in GPa) (Tsalis et al. (2015)).

$$
\tilde{C}^{\text {eff I }}=\left(\begin{array}{cccccc}
128.087 & 61.306 & 59.424 & 0 & 0 & 6.379 \\
61.306 & 121.102 & 57.853 & 0 & 0 & -0.330 \\
59.424 & 57.853 & 144.373 & 0 & 0 & 1.361 \\
0 & 0 & 0 & 35.053 & 2.509 & 0 \\
0 & 0 & 0 & 2.509 & 36.950 & 0 \\
6.379 & -0.330 & 1.361 & 0 & 0 & 35.518
\end{array}\right)
$$




\subsection{Matrix reinforced by wavy layers}

The second example presented in this paper is a matrix with wavy multilayered inclusions (see Fig. 3), made of the isotropic materials with properties depicted in Tables 9 and 10 and a waviness value 0.20 . We recall that the waviness is defined as the ratio of the heigh to the length of the wave. The volume fraction of the reinforced part is $50 \%$ and the rest $50 \%$ is pure matrix, while the volume fractions in the reinforced part are $50 \%$ for both phases. We follow the same approximation as in the "chevron" examples. The main difference is located in the second step: the result of the first step of homogenization depends on the choice of the mesh width following the $x_{1}$ axis and this choice governs the second step of homogenization, where more than two phases exist.

Table 9. Matrix with wavy layers. Mechanical properties of constituents.

\begin{tabular}{|c|c|c|}
\hline Property & Inclusions & Matrix \\
\hline \hline Young Modulus (GPa) & 200.000 & 20.000 \\
\hline Poisson Ratio & 0.300 & 0.220 \\
\hline
\end{tabular}

Table 10. Matrix with wavy layers. Elastic (symmetric) matrix coefficients of the two constituents (in GPa).

\begin{tabular}{|c|c|c|}
\hline Mechanical Properties & Inclusions & Matrix \\
\hline \hline$C_{11}, C_{22}, C_{33}$ & 269.231 & 22.834 \\
\hline$C_{12}, C_{13}, C_{23}$ & 115.385 & 6.440 \\
\hline$C_{44}, C_{55}, C_{66}$ & 76.923 & 8.197 \\
\hline$C_{14}, C_{15}, C_{16}$ & 0 & 0 \\
\hline$C_{24}, C_{25}, C_{26}$ & 0 & 0 \\
\hline$C_{34}, C_{35}, C_{36}$ & 0 & 0 \\
\hline
\end{tabular}

The generalized periodicity function for the first step of the homogenization is $\varrho^{I}=x_{2}+H \sin \left(\frac{2 \pi}{L} x_{1}\right)$ with gradients $\frac{\partial \varrho^{I}}{\partial x_{1}}=2 \pi \frac{H}{L} \cos \left(\frac{2 \pi}{L} x_{1}\right)$ and $\frac{\partial \varrho^{I}}{\partial x_{2}}=1$. The generalized periodicity function for the second step of the homogenization is $\varrho^{I I}=x_{1}$ with gradients $\frac{\partial \varrho^{I I}}{\partial x_{1}}=1$ and $\frac{\partial \varrho^{I I}}{\partial x_{2}}=0$.

We consider that the length of the unit cell is $L=1$ and we choose a number of 21 points in each of which the wave curve can be considered having a specific slope with the $x_{1}$ axis. The strategy is initially to solve 21 cell problems for a unit cell made of two isotropic elastic materials and, in the sequel, to solve 1 cell problem for a unit cell made of 21 anisotropic elastic materials resulting from the first step. In Fig. 6 we see the distribution of the elastic coefficients of the material resulting from the first step of homogenization, that behaves like a monoclinic material. The coefficients from the second step of homogenization are depicted in Table 11 and correspond to an orthotropic material. We can compare them with with the results of DIPH in Table 12 (Tsalis et al. (2015)) and we observe that the second method overestimates $8 \%$ the shear coefficients, responsible for the shear stress in $x_{1} x_{2}$ - plane. In Fig. 7 we compare the effective stresses obtained by the two methods. Recalling the results for the shearing in Tsalis et al. (2015), it seems that the present method gives a more realistic effective behavior for the simple shearing.

\subsection{Matrix reinforced by wavy fibers in two directions}

Wavy fiber reinforced composite exhibit a nice mechanical behavior (see Zesers \& Krumins (2014) for applications of this type of composites). In this example we consider a three - dimensional, two - phase composite consisting of a matrix reinforced by wavy fibers in two directions (see Fig. 8 and 9), parallel to $x_{2}-$ and $x_{1}-$ axis respectively, ordered vertically to $x_{3}-$ axis and forming two successive layers of thickness $\epsilon^{I I}$ much larger than the distance $\epsilon^{I}$ between the wavy fibers. Then, the composite can be considered as a multilayered with two types of successive reinforced layers. 


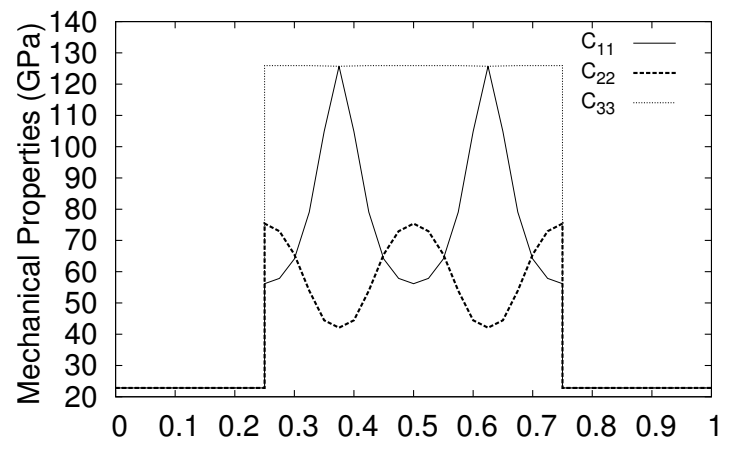

(a)

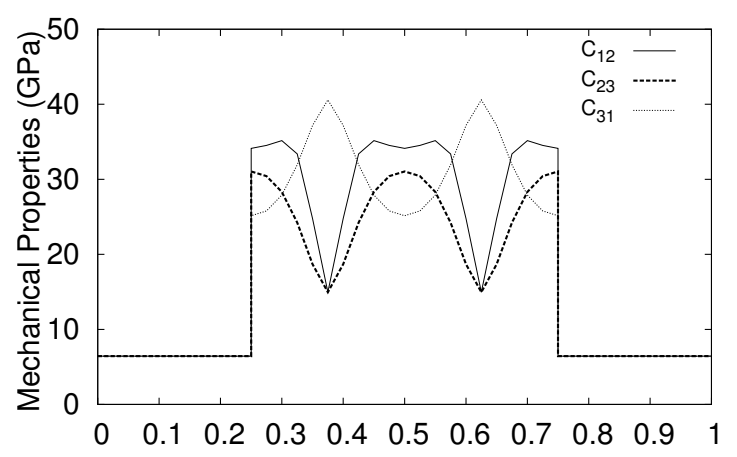

(c)

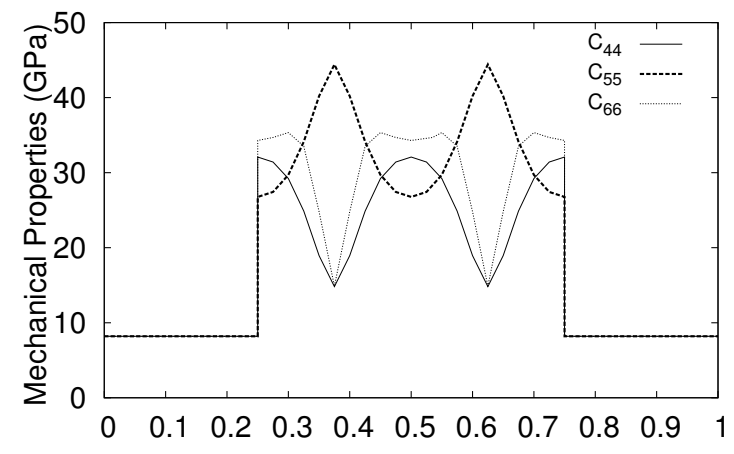

(b)

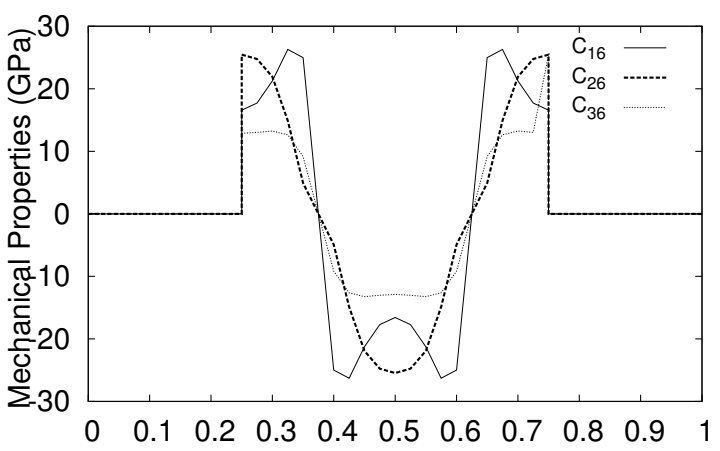

(d)

Fig. 6. Matrix with wavy layers. Distributions of coefficients (a) $C_{11}, C_{22}$ and $C_{33}$ (b) $C_{44}, C_{55}$ and $C_{66}$, (c) $C_{12}, C_{23}$ and $C_{31}$ and (d) $C_{16}, C_{16}$ and $C_{26}$ of "effective" elastic matrix along $x_{1}$ axis from the first step of homogenization.

Table 11. Matrix with wavy layers. Effective elastic matrix from the second step of homogenization (in GPa).

$$
C^{\text {eff }}=\left(\begin{array}{cccccc}
34.731 & 12.568 & 11.966 & 0 & 0 & 0 \\
12.567 & 37.739 & 12.888 & 0 & 0 & 0 \\
11.966 & 12.888 & 71.040 & 0 & 0 & 0 \\
0 & 0 & 0 & 17.044 & 0 & 0 \\
0 & 0 & 0 & 0 & 13.956 & 0 \\
0 & 0 & 0 & 0 & 0 & 12.754
\end{array}\right)
$$

Table 12. Matrix with wavy layers. Effective elastic matrix from the second step of homogenization by DIPH (in GPa).

$$
\tilde{C}^{\text {eff }}=\left(\begin{array}{cccccc}
35.299 & 11.848 & 12.006 & 0 & 0 & 0 \\
11.848 & 38.337 & 12.889 & 0 & 0 & 0 \\
11.920 & 12.851 & 71.416 & 0 & 0 & 0 \\
0 & 0 & 0 & 16.928 & 0 & 0 \\
0 & 0 & 0 & 0 & 14.800 & 0 \\
0 & 0 & 0 & 0 & 0 & 13.735
\end{array}\right)
$$




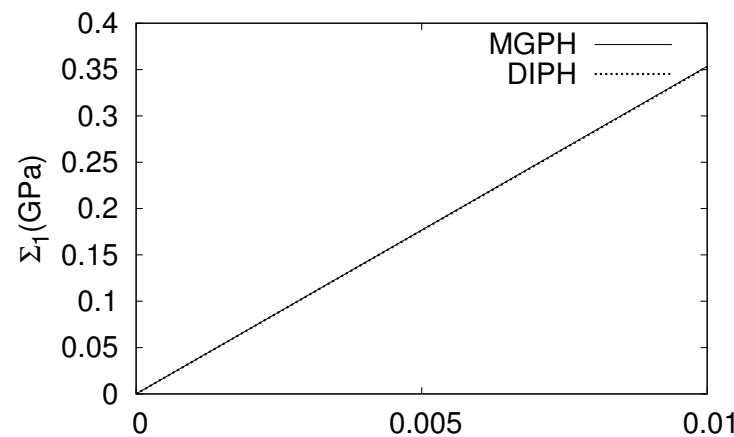

(a)

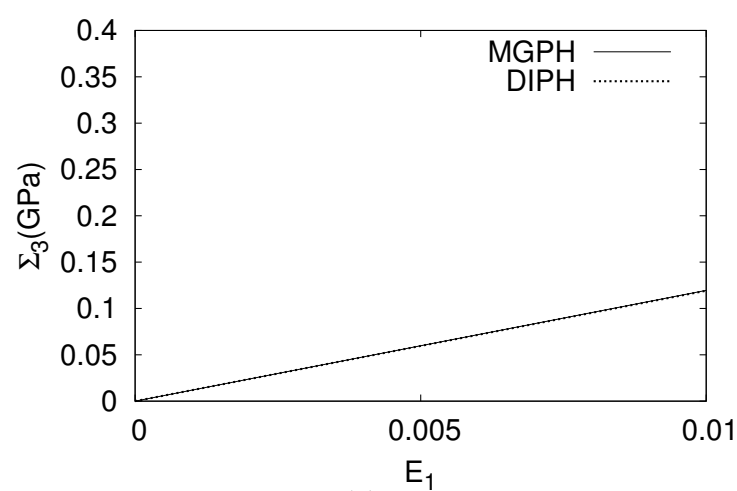

(c)

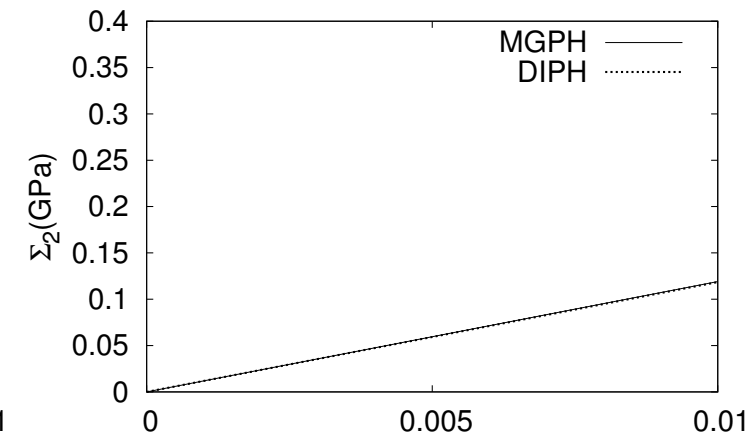

(b)

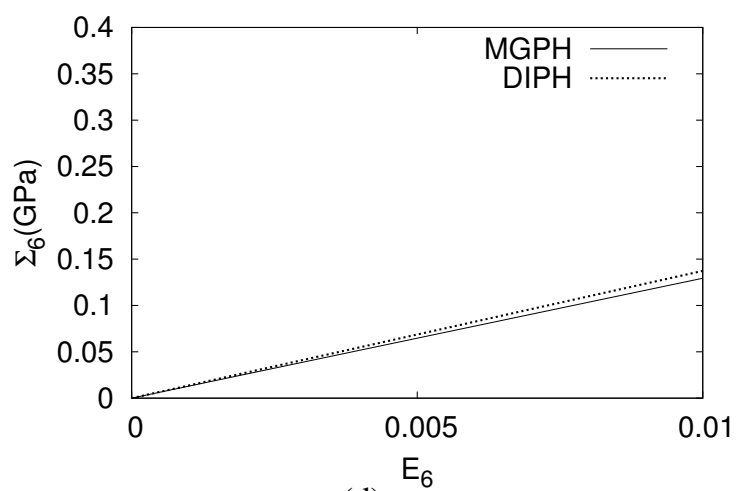

(d)

Fig. 7. Matrix with wavy layers. Macrostresses (a) $\Sigma_{1}$, (b) $\Sigma_{2}$, (c) $\Sigma_{3}$ vs macrostrain $E_{1}$ and (d) $\Sigma_{6}$ vs macrostrain $E_{6}$, where MGPH (Multi - step Generalized Periodic Homogenization) denotes the approach presented in this paper. 
Each layer consists of a matrix reinforced by fibers of waviness 0.20 . The volume fraction of fiber into each layer is $10 \%$. The first layer is piled with angle $0^{\circ}$ and the other with angle $90^{\circ}$ and so on (see Fig. 8 and 9). The properties of both matrix and fiber are shown in Tables 13 and 14.

Table 13. Matrix reinforced by wavy fibers in two directions. Mechanical properties of the two constituents.

\begin{tabular}{|c|c|c|}
\hline Property & Fiber & Matrix \\
\hline \hline Young Modulus (GPa) & 276.000 & 3.000 \\
\hline Poisson Ratio & 0.300 & 0.300 \\
\hline
\end{tabular}

Table 14. Matrix reinforced by wavy fibers in two directions. Elastic (symmetric) matrix coefficients of the two constituents (in GPa).

\begin{tabular}{|c|c|c|}
\hline Mechanical Properties & Fiber & Matrix \\
\hline \hline$C_{11}, C_{22}, C_{33}$ & 371.538 & 4.038 \\
\hline$C_{12}, C_{13}, C_{23}$ & 159.231 & 1.731 \\
\hline$C_{44}, C_{55}, C_{66}$ & 106.154 & 1.154 \\
\hline$C_{14}, C_{15}, C_{16}$ & 0 & 0 \\
\hline$C_{24}, C_{25}, C_{26}$ & 0 & 0 \\
\hline$C_{34}, C_{35}, C_{36}$ & 0 & 0 \\
\hline
\end{tabular}

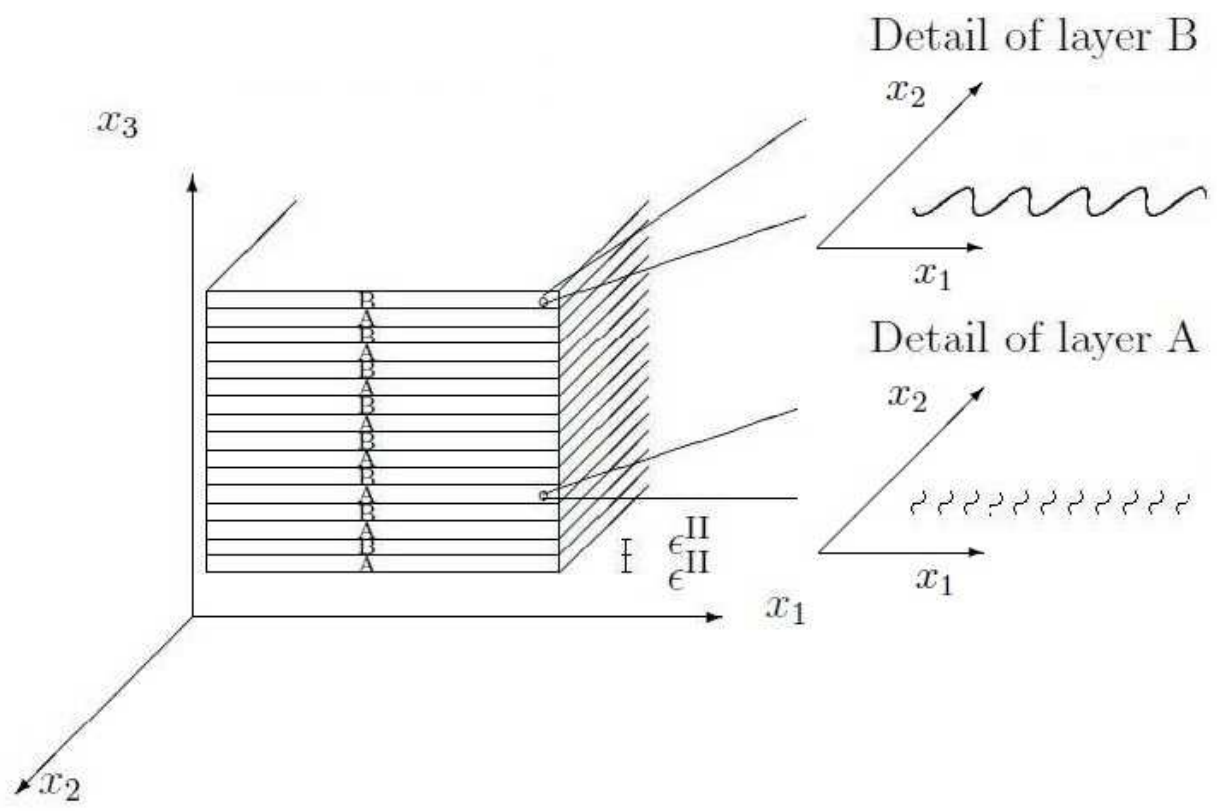

Fig. 8. Matrix reinforced by wavy fibers in two directions. Laminate stucture consists of fiber reinforced layers, piled with angles $0^{\circ}$ (layer A) and $90^{\circ}$ (layer B). For more details for layers A and B see Fig. 9.

In order to solve this problem, we follow a two - step strategy. In the first step, we compute the effective elastic modulus in the RVE of each layer, that consists of a fiber reinforced matrix cube (see Fig. 10, 11, 12 and 13). This is a three - dimensional problem that has no analytical solution, as mentioned before. In this case, we use the FEA commercial software DS Simulia Abaqus v.13 - 1 in order to solve the cell problem.

In the second step, where we will use the result of the first step as input data, we will apply the homogenization method as has been mentioned above (see "chevron" example). The volume fraction for each layer is $50.0 \%$. This is an one - 


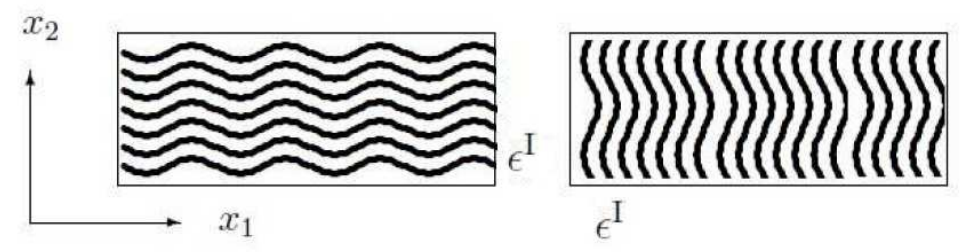

Fig. 9. Matrix reinforced by wavy fibers in two directions. Layer A (angle $0^{\circ}$ ) is shown on the left and layer B $\left(\right.$ angle $\left.90^{\circ}\right)$ is shown on the right.

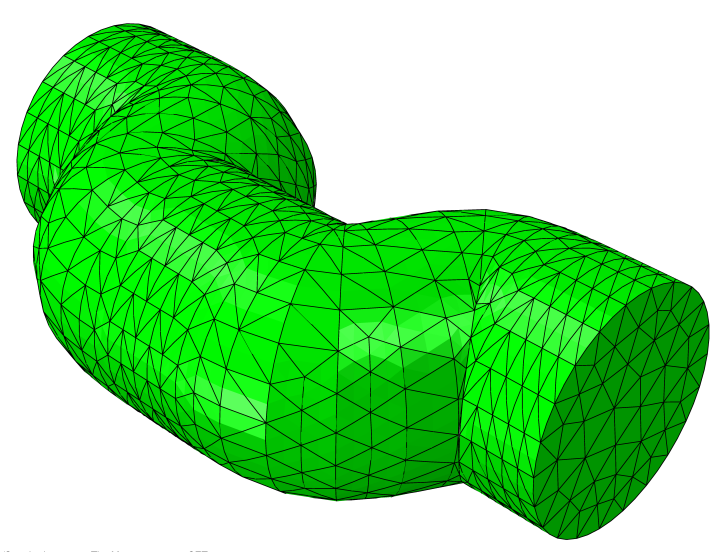

Fig. 10. Matrix reinforced by wavy fibers in two directions. Meshing of the fiber for the first step of homogenization.

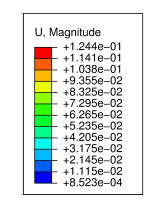

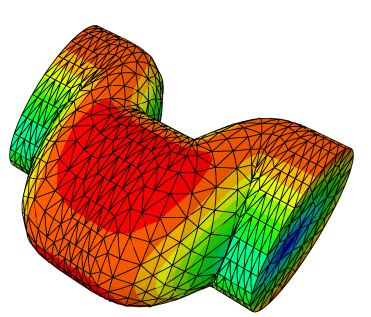

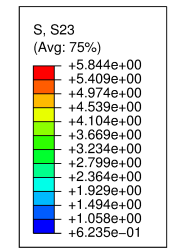

(a)

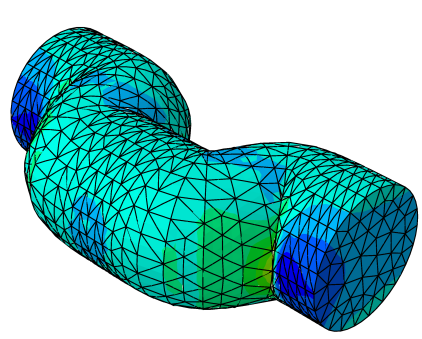

(b)

Fig. 11. Matrix reinforced by wavy fibers in two directions. (a) Displacement loading of the fiber and (b) the corresponding shear stress field of the fiber for the first step of homogenization. 
Table 15. Matrix reinforced by wavy fibers in two directions. Layer A (angle $0^{\circ}$ ). Effective elastic matrix from the first step of homogenization by DS Simulia Abaqus (in GPa).

$$
C^{\text {eff I A }}=\left(\begin{array}{cccccc}
20.320 & 2.010 & 2.260 & 0 & 0 & 0 \\
2.010 & 4.810 & 2.000 & 0 & 0 & 0 \\
2.260 & 2.000 & 5.040 & 0 & 0 & 0 \\
0 & 0 & 0 & 1.390 & 0 & 0 \\
0 & 0 & 0 & 0 & 1.620 & 0 \\
0 & 0 & 0 & 0 & 0 & 1.500
\end{array}\right)
$$

Table 16. Matrix reinforced by wavy fibers in two directions. Layer B (angle $90^{\circ}$ ). Effective elastic matrix from the first step of homogenization by DS Simulia Abaqus (in GPa).

$$
C^{\text {eff I B }}=\left(\begin{array}{cccccc}
4.810 & 2.010 & 2.000 & 0 & 0 & 0 \\
2.010 & 20.320 & 2.260 & 0 & 0 & 0 \\
2.000 & 2.260 & 5.040 & 0 & 0 & 0 \\
0 & 0 & 0 & 1.620 & 0 & 0 \\
0 & 0 & 0 & 0 & 1.390 & 0 \\
0 & 0 & 0 & 0 & 0 & 1.500
\end{array}\right)
$$

dimensional problem with two equal volume phases. The generalized periodicity function for the second step is $\varrho^{I I}=x_{3}$ with gradient $\frac{\partial \varrho^{I I}}{\partial x_{3}}=1$. The results of the first step of homogenization are shown in Tables 15 and 16 while the results of the second step are shown in Table 17.

This example illustrates the advantages of the proposed method, namely the lower computational cost, the flexibility and the great adaptativity to many cases of "hierarchical" multi - scale composites, comparing to a full FE method.

We observe that with an amount of total reinforcement $10 \%$ we managed a significant improvement of the behaviour of composite as shown in Table 22.

\section{Conclusions}

In this paper, we presented a multi - step homogenization scheme for a sequentially laminated composite made of elastic isotropic materials. The microstructure exhibits a sequence of linear or non - linear generalized periodicities. We formulated the microproblem and gave the analytical solution of it, as well as the effective coefficients at every step of the homogenization process. Moreover, we presented a combination of this method with computational homogenization techniques in order to reduce the computational cost of three - dimensional problems. The advantages of the proposed method are the lower computational cost, the flexibility and the great adaptativity to many cases of multiscale composites, comparing to a full FE method. Finally, we presented three numerical examples of engineering microstructures, namely a "chevron" like multilayer composite, a matrix reinforced by wavy layers and a matrix reinforced with wavy fibers in two directions.

Table 17. Matrix reinforced by wavy fibers in two directions. Effective elastic matrix from the second step of homogenization (in GPa).

$$
C^{\text {eff II }}=\left(\begin{array}{cccccc}
12.565 & 2.010 & 2.130 & 0 & 0 & 0 \\
2.010 & 12.565 & 2.130 & 0 & 0 & 0 \\
2.130 & 2.130 & 5.040 & 0 & 0 & 0 \\
0 & 0 & 0 & 1.505 & 0 & 0 \\
0 & 0 & 0 & 0 & 1.505 & 0 \\
0 & 0 & 0 & 0 & 0 & 1.500
\end{array}\right)
$$


Table 18. Matrix reinforced by wavy fibers in two directions. Percent improvement of the effective elastic matrix comparing to pure matrix.

$$
\left(\begin{array}{cccccc}
+211.169 \% & +16.118 \% & +23.050 \% & 0 & 0 & 0 \\
+16.118 \% & +211.169 \% & +23.050 \% & 0 & 0 & 0 \\
+23.050 \% & +23.050 \% & +24.814 \% & 0 & 0 & 0 \\
0 & 0 & 0 & +30.416 \% & 0 & 0 \\
0 & 0 & 0 & 0 & +30.416 \% & 0 \\
0 & 0 & 0 & 0 & 0 & +29.983 \%
\end{array}\right)
$$

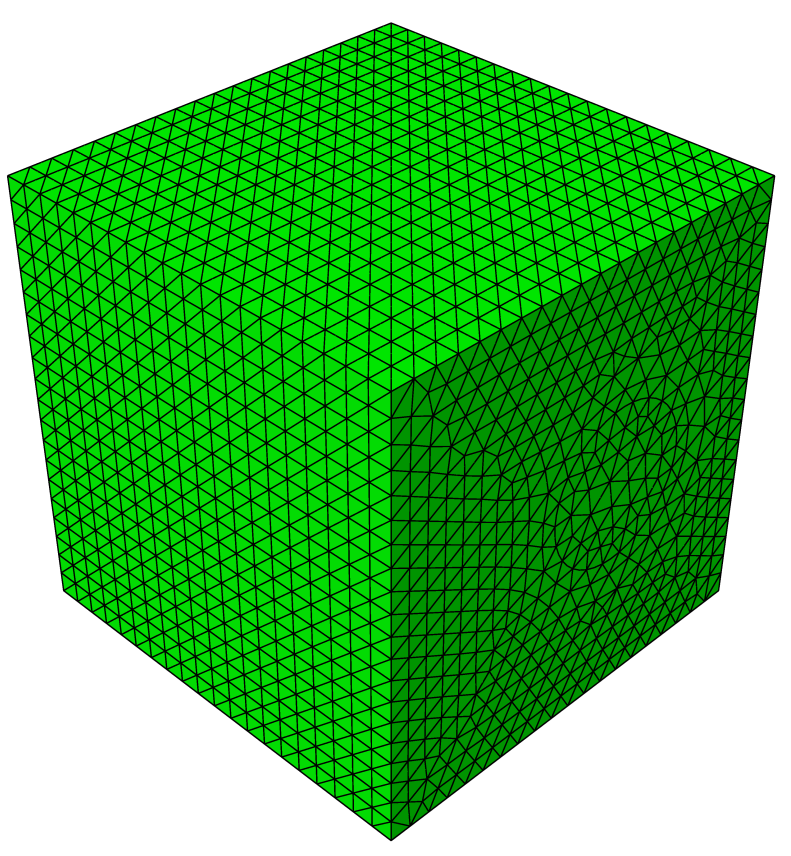

Fig. 12. Matrix reinforced by wavy fibers in two directions. Meshing of the RVE for the first step of homogenization. 


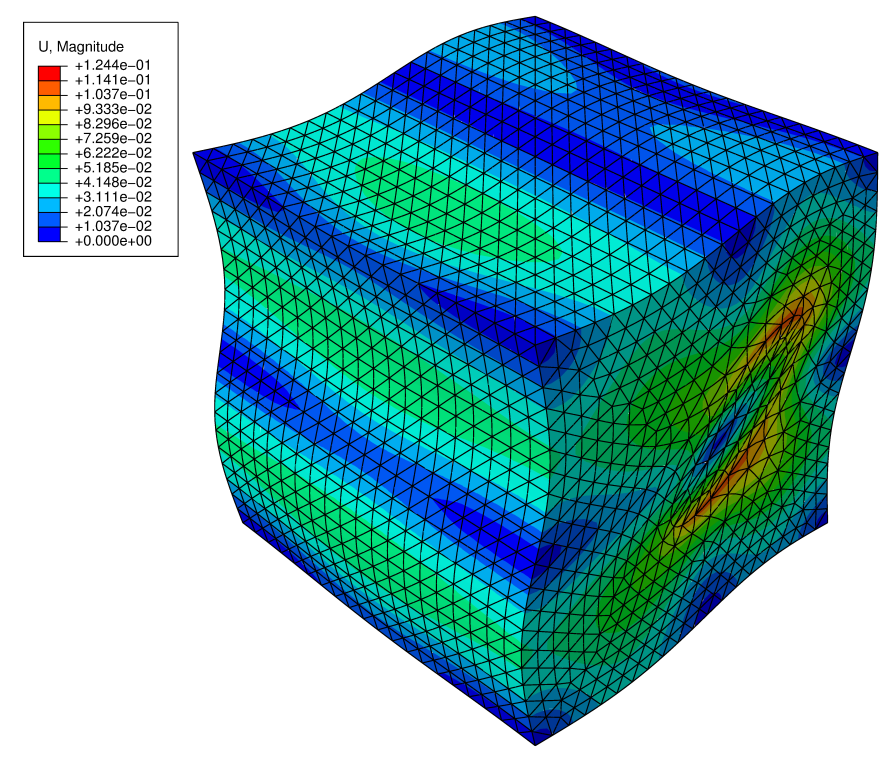

(a)

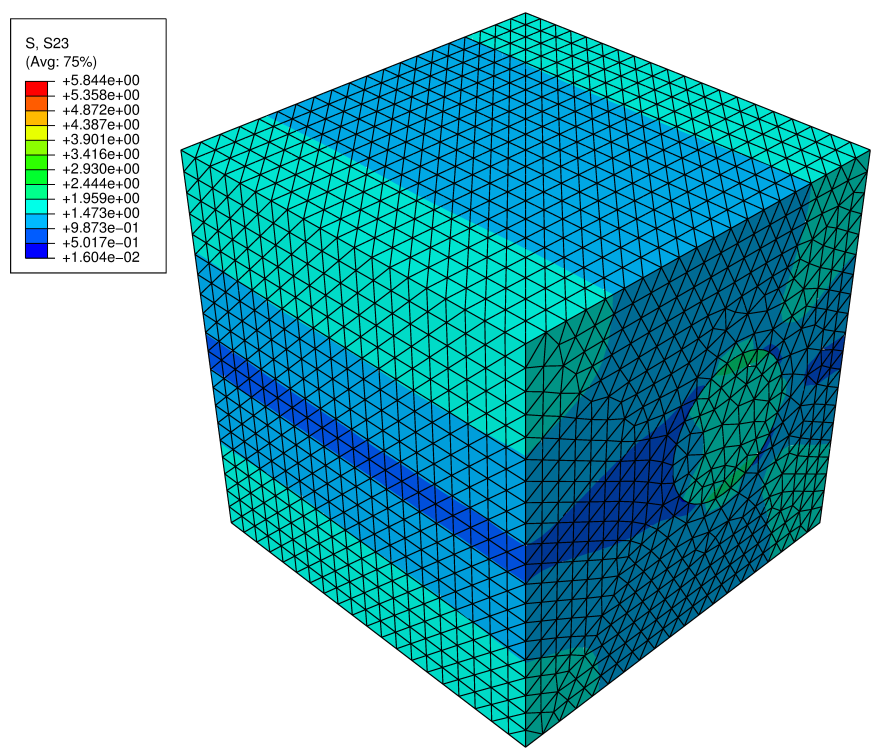

(b)

Fig. 13. Matrix reinforced by wavy fibers in two directions. (a) Displacement loading of the fiber and (b) the corresponding shear stress field of the RVE for the first step of homogenization. 
Appendix A. The system of equation (38) in matrix form. 


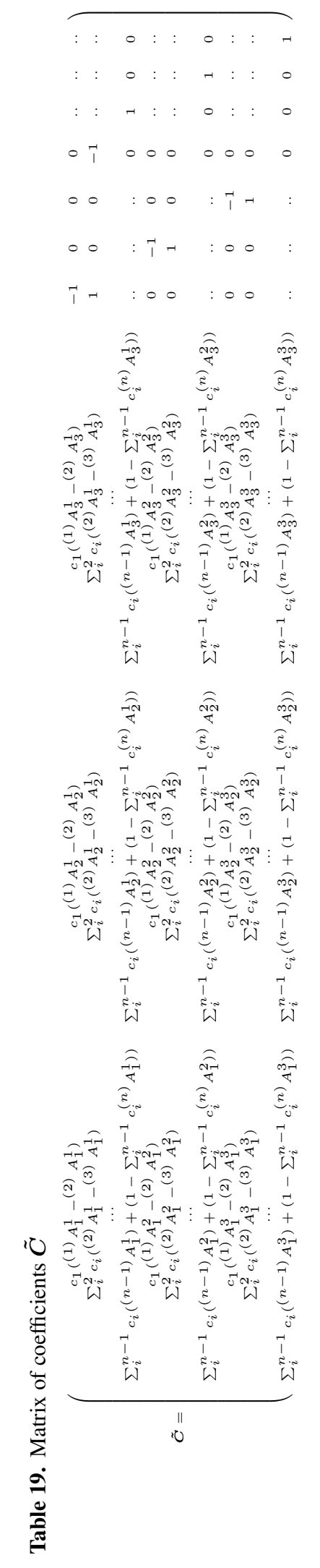




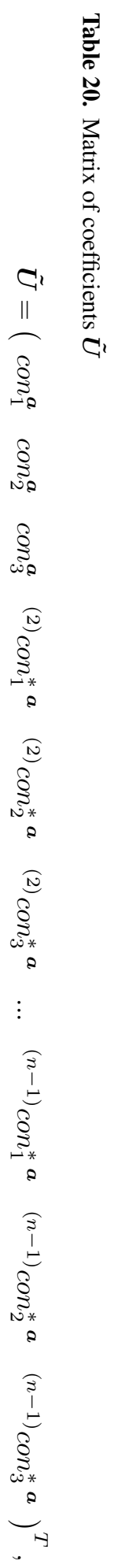




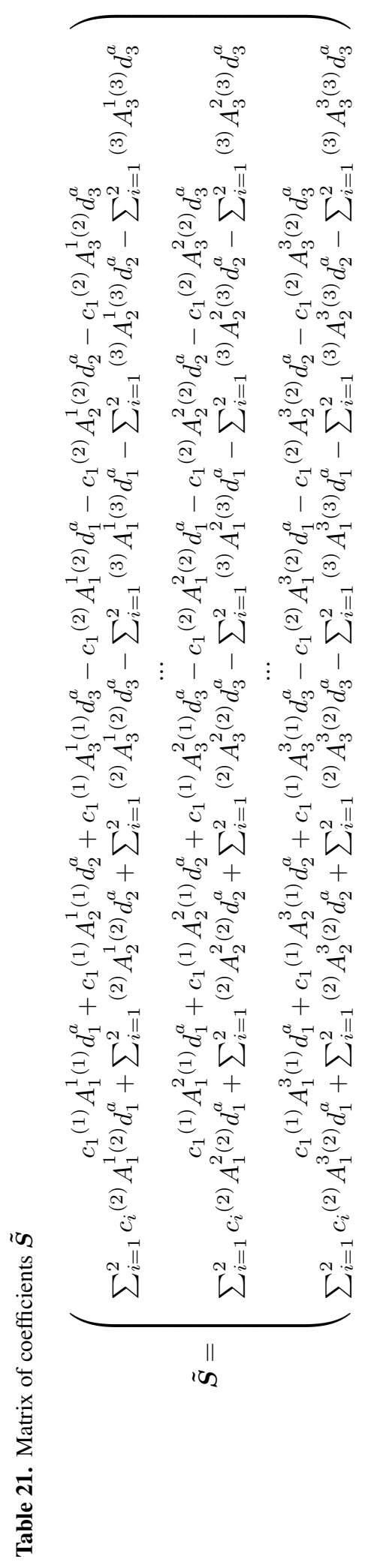


Table 22. Sets of indices for the coefficients of $a_{i j}, b_{i j}$ and $c_{i j}$ of Appendix B.

$$
\left(\begin{array}{cccccccc}
i & b & k & l & m & n & p & q \\
\hline \hline 1 & 1 & 1 & 1 & 1 & 6 & 1 & 5 \\
1 & 2 & 6 & 1 & 6 & 6 & 6 & 5 \\
1 & 3 & 5 & 1 & 5 & 6 & 5 & 5 \\
2 & 1 & 1 & 6 & 1 & 2 & 1 & 4 \\
2 & 2 & 6 & 6 & 6 & 2 & 6 & 4 \\
2 & 3 & 5 & 6 & 5 & 2 & 5 & 4 \\
3 & 1 & 1 & 5 & 1 & 4 & 1 & 3 \\
3 & 2 & 6 & 5 & 6 & 4 & 6 & 3 \\
3 & 3 & 5 & 5 & 5 & 4 & 5 & 3
\end{array}\right)
$$

\section{Appendix B. The first equation of the cell problem for the three dimensional case}

The first equation for $a=1,2,3,4,5,6$ and for $i=1,2,3$

$$
\frac{\partial}{\partial y_{i}} d_{1 i}^{a}=-\frac{\partial}{\partial y_{1}}\left(\sum_{k=1}^{3}\left(\sum_{l=1}^{3} \frac{\partial w_{k}^{a}}{\partial y_{l}} a_{k l}\right)\right)-\frac{\partial}{\partial y_{2}}\left(\sum_{k=1}^{3}\left(\sum_{l=1}^{3} \frac{\partial w_{k}^{a}}{\partial y_{l}} b_{k l}\right)\right)-\frac{\partial}{\partial y_{3}}\left(\sum_{k=1}^{3}\left(\sum_{l=1}^{3} \frac{\partial w_{k}^{a}}{\partial y_{l}} c_{k l}\right)\right)
$$

where

$$
d_{1 i}^{a}=\frac{\partial \varrho_{i}}{\partial x_{1}} C_{1 a}+\frac{\partial \varrho_{i}}{\partial x_{2}} C_{6 a}+\frac{\partial \varrho_{i}}{\partial x_{3}} C_{5 a},
$$

and for $\mathrm{j}=1,2,3$

$$
\begin{gathered}
a_{i j}=\sum_{b=1}^{3} \frac{\partial \varrho_{1}}{\partial x_{b}}\left(\frac{\partial \varrho_{j}}{\partial x_{1}} C_{k l}+\frac{\partial \varrho_{j}}{\partial x_{2}} C_{m n}+\frac{\partial \varrho_{j}}{\partial x_{3}} C_{p q}\right), \\
b_{i j}=\sum_{b=1}^{3} \frac{\partial \varrho_{2}}{\partial x_{b}}\left(\frac{\partial \varrho_{j}}{\partial x_{1}} C_{k l}+\frac{\partial \varrho_{j}}{\partial x_{2}} C_{m n}+\frac{\partial \varrho_{j}}{\partial x_{3}} C_{p q}\right), \\
c_{i j}=\sum_{b=1}^{3} \frac{\partial \varrho_{3}}{\partial x_{b}}\left(\frac{\partial \varrho_{j}}{\partial x_{1}} C_{k l}+\frac{\partial \varrho_{j}}{\partial x_{2}} C_{m n}+\frac{\partial \varrho_{j}}{\partial x_{3}} C_{p q}\right),
\end{gathered}
$$

where the combination of coefficients are shown in Table 22. 


\section{References}

A. Abd-Alla, et al. (2014). 'On problem of the non - homogeneity of an infinite orthotropic elastic cylinder'. Journal of Computational and Theoretical Nanoscience 11(4):945-952.

J. Aboudi, et al. (1999). 'Higher-order theory for functionally graded materials'. Composites: Part B 30:777-832.

G. Allaire (1992). 'Homogenization and two-scale convergence'. SIAM Journal on Mathematical Analysis 23:1482-1518.

I. Babuska (1976a). Homogenization and its application, Mathematical and computational problems, Numerical Solution of partial differential equations, III, in Proc. Third Sympos. (SYNSPADE), Univ. Maryland, 1975, pp. 89-116. Academic Press.

I. Babuska (1976b). Homogenization approach in engineering, in Computing methods in applied sciences and engineering (Second Internat. Sympos., Versailles, 1975), Part 1, pp. 137-153, Lecture Notes in Econom. and Math. Systems, Vol. 134. Springer.

R. Balokhonov, et al. (2014). 'A mesomechanical analysis of plastic strain and fracture localization in a material with a bilayer coating'. Composites: Part B 66:276-286.

A. Bensoussan, et al. (1978). Asymptotic methods for periodic structures. North Holland.

Y. Berrehili (2014). 'Lognitudinal traction of a fiber - reinforced composite beam with debonded fibers'. International Journal of Theroritical and Mathematical Physics 4(2):53-57.

V. Birman, et al. (2013). 'Strength analysis of particulate polymers'. Composites: Part B 54:278-288.

M. Briane (1990). Homogénéisation de matériaux fibrés. Ph.D. thesis, Université Pierre et Marie Curie (Paris VI).

M. Briane (1993). 'Three models of non periodic fibrous materials obtained by homogenization'. RAIRO- Modélisation mathématique et analyse numérique 27:759-775.

B. Chan \& K. Leong (2008). 'Scaffolding in tissue engineering: general approaches and tissue specific considerations'. European Spine Journal 17:467-479.

G. Chatzigeorgiou \& N. Charalambakis (2005). 'Instability analysis of non-homogeneous materials under biaxial loading'. International Journal of Plasticity 21:1970-1999.

G. Chatzigeorgiou, et al. (2012). 'Effective thermoelastic properties of composites with periodicity in cylindrical coordinates'. International Journal of Solids and Structures 49:2590-2603.

G. Chatzigeorgiou, et al. (2014). 'Unified magnetomechanical homogenization framework with application to magnetorheological elastomers'. Mathematics and Mechanics of Solids 19(2):193-211.

G. Chatzigeorgiou, et al. (2007). 'Biaxial loading of continuously graded thermoviscoplastic materials'. Computational Mechanics 39:335-355.

G. Francfort (1983). 'Homogenization and linear thermoelasticity'. SIAM Journal of Mathematical Analysis 14:696-708.

G. Francfort \& F. Murat (1986). 'Homogenization and optimal bounds in linear elasticity'. Archive for Rational mechanics and Analysis 94:307-334.

J. Freund, et al. (2014). 'Computational homogenization of regular cellular material according to classical elasticity'. Mechanics of Materials 78:56-65.

L. Gelebart (2011). 'Periodic boundary conditions for the numerical homogenization of composite tubes'. Comptes Rendus Mecanique 339:12-19.

J. Guedes \& N. Kikuchi (1990). 'Preprocessing and posprocessing for materials based on the homogenization method with adaptive finite element methods'. Computer Methods in Applied Mechanics and Engineering 83:143-198.

R. Guinovart-Diaz, et al. (2005). 'A recursive asymptotic homogenization scheme for multi-phase fiber-reinforced composites'. Mechanics of Materials 37:1119-1131.

Z. Hashin (1983). 'Analysis of composite materials: A survey'. Journal of Applied Mechanics 50:481-505.

S. Hollister \& N. Kikuchi (1992). 'A comparison of homogenization and standard mechanics analyses for periodic porous composites'. Computational Mechanics 10:73-95.

P. Jabin \& A. Tzavaras (2009). 'Kinetic decomposition for periodic homogenization problems'. SIAM Journal of Mathematical Analysis 41:360-390. 
A. Kalamkarov, et al. (2009). 'Asymptotic homogenization of composite materials and structures'. Applied Mechanics Reviews 62:030802.

A. L. Kalamkarov \& A. G. Kolpakov (1997). Analysis, design and optimization of composite structures. Wiley.

A. Katz, et al. (2015). 'Plastic strain localization in periodic materials with wavy brick-and-mortar architectures and its effect on the homogenized response'. Composites: Part B 68:270-280.

H. Khatam \& M. Pindera (2009). 'Parametric finite-volume micromechanics of periodic materials with elastoplastic phases'. International Journal of Plasticity 25:1386-1411.

R. Lakes (1993). 'Materials with structural hierarchy'. Nature 361:511-515.

A. Lebee \& K. Sab (2012). 'Homogenization of thick periodic plate: application of the Bending-Gradient plate theory to a folded core sandwich panel'. International Journal of Solids and Structures 49:2778-2792.

C. Lee \& W. Yu (2011). 'Variational asymptotic modeling of composite beams with spanwise heterogeneity'. Computers and Structures 89:1503-1511.

B. Love \& R. Batra (2006). 'Determination of effective thermomechanical parameters of a mixture of two elastothermoviscoplastic constituents'. International Journal of Plasticity 22:1026-1061.

S. Mahmoud, et al. (2014). 'The problem of wave propagation in magnetorotating orthotropic non - homogeneous medium'. Journal of Vibration and Control .

G. Milton (2002). The Theory of Composites. Cambridge University Press.

R. Moreno, et al. (2014). 'Techniques for computing fabric tensors in visualisation and processing of tensors and higher order description for multi - valued data'. Mathematics and Visualization pp. 271-292.

F. Murat \& L. Tartar (1997). H-convergence, in Topics in the mathematical modelling of composite materials, ed. by A. Cherkaev and R.V. Kohn, Progress in Nonlinear Differential Equations and their Applications, vol. 31, pp. 21-43. Birkhäuser.

U. Nackenhorst, et al. (2011). 'Computational techniques for multiscale analysis of materials and interfaces'. Lecture Notes in Applied and Computational Mechanics 57:133-167.

S. Nemat-Nasser (1999). 'Averaging theorems in finite deformation plasticity'. Mechanics of Materials 31:493-523.

S. Nemat-Nasser \& S. Hori (1999). Micromechanics: Overall Properties of Heterogeneous Materials. Elsevier, Amsterdam.

G. Nie, et al. (2011). 'Material tailoring for functionally graded hollow cylinders and spheres'. Composites Science and Technology 71:666-673.

G. Nilakantan, et al. (2008). 'Novel multi-scale modeling of woven fabric composites for use in impact studies'. 10 International LS-DYNA users conference .

M. Pindera, et al. (2012). 'Special Issue of Composites Part B: Homogenization and Micromechanics of Smart and Multifunctional Materials'. Composites: Part B 43:2493-2494.

M. Pindera, et al. (2009). 'Micromechanics of spatially uniform heterogeneous media: A critical review and emerging approaches'. Composites Part B 40:349-378.

S. Rajan, et al. (2010). 'LS-DYNA implemented multi-layer fabric material model development for engine fragment mitigation'. 11 International LS-DYNA users conference .

E. Sanchez-Palencia (1978). Non-homogeneous media and vibration theory. Lecture Notes in Physics 127, Springer Verlag.

D. Savvas, et al. (2014). 'Homogenization of random heterogeneous media with inclusions of arbitrary shape modeled by XFEM'. Computational Mechanics 54(5):1221-1235.

P. M. Suquet (1985). 'Elements of homogenization for inelastic solid mechanics'. Tech. Rep. 85-2, Université des Sciences et Techniques du Languedoc, Montpellier.

L. Tartar (1978). Nonlinear constitutive relations and homogenization in: Contemporary Developments in Continuum Mechanics and Partial Differential Equations. North-Holand.

D. Tsalis, et al. (2013). 'Homogenization of elastoplastic composites with generalized periodicity in the microstructure'. International Journal of Plasticity 51:161-187.

D. Tsalis, et al. (2012). 'Homogenization of structures with generalized periodicity'. Composites: Part B 43:2495-2512. 
D. Tsalis, et al. (2015). 'Dissipation inequality - based periodic homogenization'. Composites: Part B 76:89-104.

W. Tu \& M. Pindera (2014). 'Cohesive zone - based damage evolution in periodic materials via finite - volume homogenization'. Journal of Applied Mechanics 81(10):100-115.

Y. Wu, et al. (2014). 'Prediction of effective properties for random heterogeneous materials with extrapolation'. Archive of Applied Mechanics 84(2):247-261.

A. Zesers \& J. Krumins (2014). 'Surface properties of a hooked steel fiber and their effects on the fiber pullout and composite cracking 1. Experimental study'. Mechanics of Composite Materials 50(4):437-446.

S. Zhigang, et al. (2010). 'A quadrilateral element-based method for calculation of multi-scale temperature field'. Chinese Journal of Aeronautics 23:529-536.

D. Zhu, et al. (2014). 'Finite element modeling of ballistic impact on multi-layer Kevlar 49 fabrics'. Composites: Part B 56:254-262. 